\title{
TAUBERIAN COMPARISON THEOREMS AND HYPERBOLIC OPERATORS WITH CONSTANT COEFFICIENTS
}

\author{
YU.N. DROZHZHINOV, B.I. ZAVIALOV
}

\begin{abstract}
As Tauberian comparison theorems one usually means theorems which by a prescribed asymptotic behavior of the ratio of some integral transforms of two (generalized) functions make a conclusion on asymptotic behavior of other integral transformations of these functions. In the work we prove the comparison Tauberian function for the generalized functions whose Laplace transform have a bounded argument. In particular, examples of these functions are the kernels and the fundamental solutions of differential operators with constant coefficients hyperbolic w.r.t. a cone.
\end{abstract}

Keywords: generalized functions, Tauberian theorems, quasi-asymptotics, operators hyperbolic w.r.t. a cone.

\section{INTRODUCTION}

Theorems of Tauberian type are the theorems relating asymptotic behavior of a function (generally speaking, a generalized one) at infinity (or at zero) with the asymptotic behavior of their Laplace transform, Fourier transform or other integral transforms, of generating functions, etc., in the vicinity of zero (or of infinity). Theorems inverse to Tauberian theorems are called Abelian.

By Tauberian comparison theorems one means the theorems in which by a prescribed asymptotic behavior of quotient of integral transform for two (generalized) functions an asymptotic behavior of quotient of two other integral transforms is described. As one of the comparison function, in such theorems a so-called "admissible" generalized function is used. A typical one-dimensional Tauberian comparison theorem for the measures is the Tauberian theorem by M.V. Keldysh. We provide it in the formulation of work [1].

Theorem 1.1 (M.V. Keldysh (1951)). Let $\mu(\xi)$ and $\nu(\xi)$ be positive increasing functions defined on the half-line $(0,+\infty)$ vanishing in the vicinity of zero. Suppose that $\mu$ is differentiable and satisfies the conditinos

$$
\lim _{\xi \rightarrow \infty} \mu(\xi)=\infty, \quad \alpha<\frac{\xi \mu^{\prime}(\xi)}{\mu(\xi)}<\beta
$$

where $0<\beta<\alpha+1$. If

$$
\int_{0}^{\infty} \frac{d \mu(t)}{(t+\xi)^{[\beta]}} \sim \int_{0}^{\infty} \frac{d \nu(t)}{(t+\xi)^{[\beta]}}, \quad \xi \rightarrow+\infty,
$$

then $\mu(\xi) \sim \nu(\xi)$ as $\xi \rightarrow+\infty$. Here $[\beta]$ is the integer part of $\beta$.

Yu.N. Drozhzhinov, B.I. Zavialov, Comparison Tauberian theorems and hyperbolic operators with constant coefficients.

(c) Drozhzhinov Yu.N., Zavialov B.I. 2015.

The research is financed by the grant of Russian Scientific Foundation (project no. 14-50-00005).

Submitted July 25, 2015. 
Condition (1.1) called "M.V. Keldysh Tauberian condition" belongs to admissible conditions to be satisfied by one of the functions involving in a Tauberian comparison theorem. In monograph [2] a Tauberian comparison theorem was proven for generalized functions whose Laplace transform have a non-negative real part.

On basis of a multi-dimensional generalization of a notion of regularly varying function, in works [2], [3], 4] there was proven a series of multi-dimensional Tauberian theorems in the asymptotic scale of regularly varying functions; it included also comparison theorems. The scale of regularly varying functions was expounded in [7]. These theorems had numerous applications in spectral theory, probability theory and various models of mathematical physics [2], 8], 9]. The present paper is the continuation of work [5]. We show that a generalized function whose Laplace transform has a bounded argument is admissible. In particular, such functions are the kernels of operators hyperbolic w.r.t. a cone. We prove a Tauberian comparison theorem, in which as compared generalized functions we choose kernels and fundamental solutions of operators hyperbolic w.r.t. homogeneous cones.

\section{BASIC NOTATIONS AND SOME DEFINITIONS}

Let $\Gamma$ be a closed acute regular bodily homogeneous cone in $\mathbb{R}^{n}$ with the vertex at 0 ,

$$
\Gamma^{*}=\left[y \in \mathbb{R}^{n}:(y, t) \geq 0, t \in \Gamma\right], \quad C=\operatorname{int} \Gamma^{*}
$$

be the dual cone. Among such cones there is, for instance, the light cone of future

$$
\Gamma=\bar{V}_{n}^{+}=\left[t=\left(t_{0}, \bar{t}\right) \in \mathbb{R}^{n+1}: t_{0} \geqslant|\bar{t}|\right]
$$

and the positive quadrantal cone

$$
\overline{\mathbb{R}}_{+}^{n}=\left\{t \in \mathbb{R}^{n}: t_{j} \geqslant 0, j=1, \ldots, n\right\} .
$$

These two cones are self-adjoint.

By $S^{\prime}(\Gamma)$ we denote the space of tempered functions with supports in cone $\Gamma$. The Laplace transform of generalized functions $f(t) \in S^{\prime}(\Gamma)$ defined by the formula

$$
L[f(t)] \equiv \tilde{f}(z)=\left(f(t), e^{i(z, t)}\right), \quad z=x+i y, \quad x \in \mathbb{R}^{n}, \quad y \in C,
$$

makes an isomorphism of convolution algebra $S^{\prime}(\Gamma)$ onto algebra $H\left(T^{C}\right)$ of functions holomorphic in the tubular domain $T^{C}=\mathbb{R}^{n}+i C$ with the polynomial rate of growth at the boundary:

$$
|\widetilde{f}(z)| \leqslant M \frac{(1+|z|)^{a}}{\Delta_{C}^{b}(y)}, \quad z=x+i y \in T^{C},
$$

for some $M, a, b$ depending on $\widetilde{f}$. Here $\Delta_{C}(y)$ is the distance from $y$ to the boundary of cone $C$. Generalized function $f(t) \in S^{\prime}(\Gamma)$ is called spectral function for $\widetilde{f}(z)$.

The Cauchy kernel of tubular domain $T^{C}$ is introduced by the formula

$$
\mathcal{K}_{C}(z)=L\left[\Theta_{\Gamma}(t)\right](z) \text { for } z \in T^{C},
$$

where $\Theta_{\Gamma}(t)$ is the characteristic function of cone $\Gamma$. By the regularity of $\Gamma$, Cauchy kernel is the divisor of unity in algebra $H\left(T^{C}\right)$. This is why generalized functions $\Theta_{\Gamma}^{\alpha}(t)$ defined by the formula

$$
\mathcal{K}_{C}^{\alpha}(z)=L\left[\Theta_{\Gamma}^{\alpha}(t)\right], \quad-\infty<\alpha<\infty,
$$

form convolution Abelian group: $\Theta_{\Gamma}^{\alpha} \star \Theta_{\Gamma}^{\beta}=\Theta_{\Gamma}^{\alpha+\beta}$. Generalized function $f^{(-\alpha)}=\Theta_{\Gamma}^{\alpha} \star f$ is called primitive (or derivative for $\alpha<0$ ) of a generalized function $f \in S^{\prime}(\Gamma)$ of order $\alpha$ w.r.t. cone $\Gamma$. In particular, $\mu(t)=\int_{(t-\Gamma) \cap \Gamma} d \mu(t)$ is the primitive of measure $d \mu(t)$ w.r.t. cone $\Gamma$. And the half derivative $\alpha=-\frac{1}{2}$ w.r.t. the cone of future is the D'Alambert operator

$$
\Theta_{V_{+}^{3}}^{-\frac{1}{2}}(t)=\operatorname{const}\left(\partial_{0}^{2}-\Delta\right) \delta(t), \quad t=\left(t_{0}, t_{1}, t_{2}, t_{3}\right),
$$


By $\mathcal{A}(\Gamma)$ we denote the set of proper linear automorphisms of cone $\Gamma$ so that

$$
U \in \mathcal{A}(\Gamma): \quad U \Gamma \subset \Gamma, \quad \operatorname{det} U=J>0 .
$$

Respectively, operator $V=\left(U^{T}\right)^{-1}$ defines an automorphism of cone $\Gamma^{*}$ and hence $V \subset \mathcal{A}\left(\Gamma^{*}\right)$. We note that

$$
\Theta_{\Gamma}^{\alpha}(U t)=J^{\alpha-1} \Theta_{\Gamma}^{\alpha}(t)
$$

It is easy to see for the light cone of future $\bar{V}_{n}^{+}$we have $\Theta_{V_{n}^{+}}^{\sigma}(t) \in L_{2}^{\text {loc }}$ as $\sigma>1-\frac{1}{n+1}$. Similarly, for the positive octant $\mathbb{R}_{+}^{n}$ as $\sigma>\frac{1}{2}$ we have $\Theta_{\mathbb{R}_{+}^{n}}^{\sigma} \in L_{2}^{\text {loc }}$. Suppose we are given a family $\left\{U_{k} \in \mathcal{A}(\Gamma), k \in I\right\}$, where the $+\infty$ is the accumulation potin for the set of indices $I$.

Definition. A complex-valued generalized function $u(t) \in S^{\prime}(\Gamma)$ is called $q$-completely admissible for family $\left\{U_{k} \in \mathcal{A}(\Gamma), k \in I\right\}$ if

1. $u^{(-q)}(t)$ is locally integrable function;

2. There exists $t_{0} \in \operatorname{int} \Gamma$ such that

$$
\Phi_{k}(t)=\frac{u^{(-q)}\left(U_{k} t\right)}{u^{(-q)}\left(U_{k} t_{0}\right)} \stackrel{t \in K}{\rightrightarrows} \gamma_{q}(t), \quad k \rightarrow+\infty
$$

where $K$ is an arbitrary compact in int $\Gamma$, and function $\gamma_{q}(t) \neq 0$ is continuous in int $\Gamma$;

3. There exists $k_{0}$ such that $\left|\Phi_{k}(t)\right| \leqslant \psi(t)$ as $k>k_{0}$ and $t \in \operatorname{int} \Gamma$, where $\psi(t)$ is a tempered function in $\Gamma$.

Definition 2.1. A generalized function $u(t) \in S^{\prime}(\Gamma)$ is called $q$-admissible for cone $\Gamma$ if for each family $\left\{U_{k} \in \mathcal{A}(\Gamma), k \in I\right\}$ there exists a subsequence $\left\{U_{k_{m}}, m \rightarrow \infty, k_{m} \in I\right\}$, w.r.t. which generalized function $u(t)$ is $q$-completely admissible.

We observe that if function $u(t)$ is $q$-admissible, it is also $(q+n)$-admissible $n=1,2, \ldots$.

In works [2, [3], [4], [5] admissible and completely admissible functions were introduced, the examples were given, and some sufficient condition for $q$-admissibility were provided. Such functions cover an important class of functions whose Laplace transform has a bounded argument. In particular, the kernels of passive operators (their Laplace transform have a nonnegative real part) belong to this class.

Definition 2.2. We shall say that a complex-valued continuously differentiable function $u(t) \in S^{\prime}(\Gamma)$ satisfies generalized Keldysh condition if there exists a set of vectors $\mathcal{O} \subset \Gamma$

$$
U \mathcal{O} \subset \mathcal{O}, \quad \forall U \in \mathcal{A}(\Gamma) ; \quad \operatorname{Lin}(\mathcal{O})=\mathbb{R}^{n}
$$

such that the set

$$
D=\left\{\xi \in \mathbb{C}: \xi=\frac{(\ell, t)(\ell, \operatorname{grad} u(t))}{u(t)}, t \in \operatorname{int} \Gamma,|\ell|=1, \ell \in \mathcal{O}\right\}
$$

is bounded and $\bar{D} \subset\{\xi \in \mathbb{C}: \operatorname{Re} \xi>-1\}$.

It was shown in work [5] that functions satisfying generalized Keldysh condition are zeroadmissible for cone $\Gamma$ if $\Gamma$ is a positive quadrantal angle or the light cone of future. The following theorem was proven.

Theorem 2.1. Let $u(t) \in S^{\prime}(\Gamma)$ be real and

$$
|\arg \widetilde{u}(z)| \leqslant \frac{\pi}{2} m, \quad z \in T^{C},
$$

where $m$ is an integer. If

$$
\Theta_{\Gamma}^{\sigma}(t) \in L_{2}^{l o c},
$$

then $u(t)$ is $[(2+\sigma) m]$-admissible for $\Gamma$. 


\section{TAUBERIAN COMPARISON THEOREMS}

In work [5], a rather general Tauberian theorem for holomorphic functions of bounded argument was proven.

Theorem 3.1. Let $f(t) \in S^{\prime}(\Gamma)$ and $|\arg \tilde{f}(z)| \leqslant \frac{\pi}{2} m, z \in T^{C}$. Suppose also that we are given a sequence of numbers $\left\{\rho_{k}, r \in I\right\}$ and a family $\left\{U_{k} \in \mathcal{A}(\Gamma), k \in I\right\}$. If there exists a domain $\Omega \subset C$ such that

$$
\frac{1}{J_{k} \rho_{k}} \tilde{f}\left(V_{k} y\right) \longrightarrow \widetilde{h}(y), \quad k \rightarrow \infty, \quad y \in \Omega,
$$

then for each $q \geqslant(2+\sigma) m$, where $\sigma$ is such that $\Theta_{\Gamma}^{\sigma} \in L_{2}^{\text {loc }}$,

$$
\frac{1}{J_{k}^{q} \rho_{k}} f^{(-q)}\left(U_{k} t\right) \stackrel{t \in K}{\rightrightarrows} \gamma_{q}(t), \quad k \rightarrow \infty,
$$

where $\gamma_{q}$ is continuous, and $K$ is an arbitrary compact set in int $\Gamma$. Moreover, $\widetilde{\gamma}_{q}(i y)=$ $\widetilde{h}(i y) \mathcal{K}_{C}^{q}(i y)$ and the estimate

$$
\left|\frac{1}{J_{k}^{q} \rho_{k}} f^{(-q)}\left(U_{k} t\right)\right| \leqslant \psi(t), \quad k>k_{0},
$$

holds true for some $k_{0}$, where $\psi(t)$ has a polynomial growth in $\Gamma$.

We note that in accordance with Theorem 2.1. function $\gamma_{q}(t)$ coincides with the function determined by the condition of $q$-complete admissibility, see 2.1. The proof employs essentially the general Tauberian theorem of work [2] and a special estimate of Cauchy kernel for an acute regular cone [6]. We note that in the application it is usually assumed that family $\left\{U_{k}, k>0\right\}$ is a real continuous multiplicative group of linear automorphisms of cone $\Gamma$ such that $\left\{U_{k}(t), k>0\right\}$ defines phase trajectories of a simplest dynamical system, the real parts of all the eigenvalues of the generator for this group are of the same sign [10, 11].

The main aim of this work is to prove Tauberian comparison theorem for holomorphic functions of bounded argument.

Theorem 3.2. Let $f(t) \in S^{\prime}(\Gamma), \quad|\arg \widetilde{f}(z)| \leqslant \frac{\pi}{2} m$ as $z \in T^{C}$, and function $u(t)$ is $q^{-}$ admissible for $\Gamma$, at that, $q \geqslant(2+\sigma) m$ and $\Theta_{\Gamma}^{\sigma} \in L_{2}^{\text {loc }}$, while $\left\{U_{k}, k \in I\right\}$ is a given family of linear automorphisms of cone $\Gamma$. If there exists a domain $\Omega \subset C$ such that

$$
\frac{\widetilde{f}\left(V_{k} y\right)}{\widetilde{u}\left(V_{k} y\right)} \longrightarrow \widetilde{p}(y), \quad k \rightarrow \infty, k \in I, \quad y \in \Omega,
$$

then

$$
\frac{f^{(-q)}\left(U_{k} t\right)}{u^{(-q)}\left(U_{k} t\right)} \stackrel{t \in K}{\rightrightarrows} \frac{\gamma_{q}(t) * p(t)}{\gamma_{q}(t)}, \quad k \rightarrow \infty
$$

where $K$ is arbitrary compact set in int, $\Gamma, \widetilde{p}(z)=L[p(t)]$, and function $\gamma_{q}(t)$ is defined by formula (3.1).

Доказательство. Suppose that condition (3.2) is satisfied, while (3.3) is not, i.e., for some numbers $m_{0}, \varepsilon_{0}>0$ there exists a subsequence

$$
\left\{U_{k_{m}} \subset \mathcal{A}(\Gamma), \quad k_{m} \in I, \quad m \rightarrow \infty\right\}
$$

and a sequence of points $\left\{t_{m} \in K\right\}$ such that

$$
\left|\frac{f^{(-q)}\left(U_{k_{m}} t_{m}\right)}{u^{(-q)}\left(U_{k_{m}} t_{m}\right)}-\frac{\gamma_{q}(t) * p(t)}{\gamma_{q}(t)}\right|_{t=t_{m}} \mid>\varepsilon_{0}, \quad m>m_{0} .
$$


Letting $\rho_{k}=\frac{1}{J_{k}^{q}} u^{(-q)}\left(U_{k} t_{0}\right)$, in view of $q$-admissibility of function $u(t)$ for some subsequence $\left\{U_{k_{m}^{\prime}}, m^{\prime} \rightarrow \infty\right\}$ we have

$$
\frac{1}{J_{k_{m}}^{q} \rho_{k_{m}}} u^{(-q)}\left(U_{k_{m}} t\right)=\frac{u^{(-q)}\left(U_{k_{m}} t\right)}{u^{(-q)}\left(U_{k_{m}} t_{0}\right)} \stackrel{t \in K}{\rightrightarrows} \gamma_{q}(t), \quad m \rightarrow \infty .
$$

Here we have omitted primes for $m$ that does not spoils the generality in the proof. Moreover, for $y \in \Omega$ we have

$$
\frac{\widetilde{f}\left(V_{k_{m}} y\right)}{J_{k_{m}} \rho_{k_{m}}}=\frac{\widetilde{f}\left(V_{k} y\right)}{\widetilde{u}\left(V_{k} y\right)} \frac{J_{k_{m}}^{q-1} \widetilde{u}\left(V_{k_{m}} y\right)}{u\left(U_{k_{m}} t_{0}\right)} \rightarrow \widetilde{p}(y) \mathcal{K}_{C}^{(-q)}(i y) \widetilde{\gamma}_{q}(i y) .
$$

Here we have taken into consideration that by $(3.2)$ the first fact in the left hand side tends to $\widetilde{p}(i y)$, while, by Lemma 1 in [2, Sect. 5.2], the second tends to $\mathcal{K}_{C}^{(-q)}(i y) \widetilde{\gamma}_{q}(i y)$. In accordance with Tauberian theorem 3.1 for $m \rightarrow \infty$ we have

$$
\frac{1}{J_{k_{m}}^{q} \rho_{k_{m}}} f^{(-q)}\left(U_{k_{m}} t\right)=\frac{f^{(-q)}\left(U_{k} t\right)}{u^{(-q)}\left(U_{k} t\right)} \frac{u^{(-q)}\left(U_{k_{m}} t\right)}{u^{(-q)}\left(U_{k_{m}} t_{0}\right)} \stackrel{t \in K}{\rightrightarrows} p(t) \gamma_{q}(t) .
$$

Comparing relations (3.5), (3.7), and (3.4), we arrive at the contradiction that completes the proof.

Theorem 3.1 and Theorem 3.2 allow us to study the quasi-asymptotic of the kernels of differential operators with constant coefficients hyperbolic w.r.t. cone $C$ and of their fundamental solutions.

Let $U=\left\{U_{k}, k>0\right\}$ be a multiplicative one-parametric group of linear automorphisms of cone $\Gamma$ so that $U_{k} \in \mathcal{A}(\Gamma)$. At that, we assume that $\left\{U_{k} t, k>0, t \in \Gamma\right\}$ define phase trajectories of the dynamical system

$$
\frac{d t(\tau)}{d \tau}=A t, \quad \tau=\ln k,
$$

in which all the eigenvalues of matrix $A$ being the generator of this group are positive. We recall the definition of quasi-asymptotics of a generalized function in the scale of regularly varying functions.

Let $f(t) \in S^{\prime}(\Gamma)$ and $\rho(k)$ be a regularly varying function. We shall say that $f$ possesses a quasi-asymptotics at zero (at infinity) w.r.t. $\rho(k)$ by group $U_{k} \in \mathcal{A}(\Gamma)$ if for each test function $\psi(t) \in S(\Gamma)$ and some $g \in S^{\prime}(\Gamma), g \not \equiv 0$,

$$
\frac{1}{\rho(k)}\left(f\left(U_{\frac{1}{k}} t\right), \psi(t)\right) \underset{k \rightarrow \infty}{\longrightarrow}(g(t), \psi(t)) \quad\left(\frac{1}{\rho(k)}\left(f\left(U_{k} t\right), \psi(t)\right) \underset{k \rightarrow \infty}{\longrightarrow}(g(t), \psi(t))\right),
$$

for details see [11].

We consider a differential operator with constant coefficients of $m$-th order

$$
Q(\partial)=\sum_{|\alpha| \leqslant m} a_{\alpha} \partial^{\alpha}, \quad \sum_{|\alpha|=m}\left|a_{\alpha}\right| \neq 0 .
$$

Operator $Q(\partial)$ is called hyperbolic w.r.t. cone $C$ if there exists a point $y_{0} \in \mathbb{R}^{n}$ such that $Q\left(y_{0}-i z\right) \neq 0, z \in T^{C}$. We let $P(\partial)=Q\left(y_{0}+\partial\right)$ so that $P(-i z)=Q\left(y_{0}-i z\right) \neq 0$ and therefore, it is a hyperbolic polynomial.

Lemma 3.1. A hyperbolic polynomial $P(-i z)$ non-zero in $T^{C}$ has a bounded argument there.

The proof was provided in [2].

Let $P_{i}(\partial), i=1,2$, by operators hyperbolic w.r.t. $C$ and $\mathcal{E}_{i}(t) \in S^{\prime}(\Gamma)$ be their fundamental solutions so that for the Laplace transform we have

$$
P_{i}(-i z) \widetilde{\mathcal{E}}_{i}(-i z)=1, \quad i=1,2, \quad z \in T^{C} .
$$


In particular, it implies that the Laplace transform of fundamental solutions for hyperbolic operators have a bounded argument in $T^{C}$ and moreover, for the group $\left\{V_{k} \in \mathcal{A}(C), k>0\right\}$ (more precisely, for the phase trajectories defined by this group),

$$
\frac{\widetilde{\mathcal{E}}_{1}\left(V_{k} y\right)}{\widetilde{\mathcal{E}}_{2}\left(V_{k} y\right)}=\frac{P_{2}\left(V_{k} y\right)}{P_{1}\left(V_{k} y\right)}, \quad y \in C .
$$

Employing now Theorem 3.2, we obtain the following Tauberian comparison theorem for the fundamental solutions of hyperbolic operators.

Theorem 3.3. Let $P_{i}(\partial), i=1,2$, by operators hyperbolic w.r.t. cone $C=\operatorname{int} \Gamma^{*}$ and $\mathcal{E}_{i}(t) \in$ $S^{\prime}(\Gamma)$ be their fundamental solutions. Let also $\sigma$ be such that $\Theta_{\Gamma}^{\sigma}(t) \in L_{2}^{\text {loc }}$. If for some domain $\Omega \in C$ and group $\left\{V_{k} \in \mathcal{A}(C), k>0\right\}$

$$
\frac{P_{2}\left(V_{k} y\right)}{P_{1}\left(V_{k} y\right)} \stackrel{y \in \Omega}{\rightrightarrows} \widetilde{p}(y), \quad k \rightarrow \infty
$$

then for some $q$

$$
\frac{\mathcal{E}_{1}^{(-q)}\left(U_{k} t\right)}{\mathcal{E}_{2}^{(-q)}\left(U_{k} t\right)} \stackrel{t \in K}{\rightrightarrows} \frac{\gamma_{q}(t) * p(t)}{\gamma_{q}(t)}, \quad k \rightarrow \infty
$$

Here $K$ is an arbitrary compact set in int $\Gamma, \widetilde{p}(z)=L[p(t)]$, and function $\gamma_{q}(t)$ is defined by formula (3.1), in which $u(t)$ should be replaced by $P_{1}(t)$. At that, $q>(2+\sigma) m$, where $m$ is determined by Theorem 2.1.

Example. We consider differential operators hyperbolic w.r.t. cone $\overline{\mathbb{R}}_{+}^{2}$

$$
P_{1}(\partial)=\frac{\partial^{2}}{\partial t_{1} \partial t_{2}} \delta\left(t_{1}, t_{2}\right) *, \quad P_{2}(\partial)=\left(\frac{\partial^{2}}{\partial t_{1}^{2}}+2 \frac{\partial^{2}}{\partial t_{1} \partial t_{2}}+\frac{\partial^{2}}{\partial t_{2}^{2}}\right) \delta\left(t_{1}, t_{2}\right) * .
$$

In $T^{C}=\mathbb{R}^{2}+i \mathbb{R}_{+}^{2}$, the Laplace transform of their kernels reads as $\widetilde{P}_{1}(-i z)=-z_{1} z_{2}$ and $\widetilde{P}_{2}(-i z)=-\left(z_{1}^{2}+2 z_{1} z_{2}+z_{2}^{2}\right)$ does not vanish (here $\left.m=4\right)$. We have

$$
\widetilde{P}_{1}(y)=y_{1} y_{2}, \quad \gamma_{1}(t)=\Theta_{\mathbb{R}_{+}^{2}}(t), \quad \widetilde{P}_{2}(y)=y_{1}^{2}+2 y_{1} y_{2}+y_{2}^{2},
$$

where $\Theta_{\mathbb{R}_{+}^{2}}(t)$ is a fundamental solution of operator $P_{1}$ up to a multiplicative constant. As phase trajectories we use the rays leaving the origin,

$$
U_{k} t=k t, \quad V_{k} y=\frac{1}{k} y, \quad \frac{d t}{d \tau}=E t, \quad \tau=\ln k,
$$

i.e., the generator of the group is the unit matrix. By relation (3.12) (up to a constant) we have

$$
1=c \mathcal{E}_{2}(t)\left[t_{2} \delta\left(t_{1}\right)+2 \delta\left(t_{1}, t_{2}\right)+t_{1} \delta\left(t_{2}\right)\right] .
$$

We observe that this relation is formal since one can not to divide the unit by the factor from the left, one first should take appropriate primitive w.r.t. cone $\overline{\mathbb{R}}_{+}^{2}$. In accordance with the theorem, it is sufficient to take the ninth primitive (in fact, the third is enough). Other numerous applications of Tauberian theorems can be found in Chapter IV of monograph [2].

\section{BIBLIOGRAPHY}

1. A.G. Kostyuchenko, I.S. Sargsyan. Distribution of eigenvalues. Self-adjoint ordinary differential operators. Nauka, Moscow (1979). (in Russian).

2. V.S. Vladimirov, Yu.N. Drozhzhinov, B.I. Zav'yalov. Tauberian theorems for generalized functions. Nauka, Moscow (1986). [Mathematics and Its Applications (Soviet Series), 10. Kluwer Academic Publishers, Dordrecht (1988).] 
3. Yu.N. Drozhzhinov, B.I. Zav'yalov. Multidimensional Tauberian comparison theorems for generalized functions in cones // Matem. Sborn. 126:4, 515-542 (1985). [Math. USSR. Sb. 54:2, 499-524 (1986).]

4. Yu.N. Drozhzhinov, B.I. Zav'yalov. Multidimensional Abelian and Tauberian comparison theorems // Matem. Sborn. 180:9, 1234-1259 (1989). [Math. USSR. Sb. 68:1. 85-110 (1991).]

5. Yu.N. Drozhzhinov, B.I. Zav'yalov. Multidimensional Tauberian comparison theorems for holomorphic functions of bounded argument// Izv. AN SSSR. Ser. Matem. 55:6, 1139-1155. [Math. USSR. Izv. 39:3, 1097-1112 (1992).]

6. V.S. Vladimirov. Generalized functions in mathematical physics. Nauka, Moscow (1979). [Mir Publishers, Moscow (1979).]

7. E. Seneta. Regularly varying functions. Springer-Verlag, Berlin (1976).

8. A.L. Yakimiv. Probabilistic applications of Tauberian theorems. Fizmatlit, Moscow (2005). [Modern Probability and Statistics. VSP, Leiden (2005).]

9. K.Kh. Boimatov. Multidimensional spectral asymptotics for elliptic operators in domains that satisfy the cone condition // Dokl. AN SSSR. 316:1, 14-18 (1991). [Soviet Math. Dokl. 43, 4-8 (1991).]

10. Yu.N. Drozhzhinov, B.I. Zav'yalov Generalized functions asymptotically homogeneous along the unstable degenerated node // Vestn. Samar. Gos. Tekhn. Univ. Ser. Fiz.-Mat. Nauki. 1(22), 68-82 (2011). (in Russian).

11. Yu.N. Drozhzhinov, B.I. Zav'yalov. Distributions asymptotically homogeneous along the trajectories determined by one-parameter groups // Izv. RAN. Ser. Matem. 76:3, 39-92 (2012). [Izv. Math. 76:3, 466-516 (2012).]

Yurii Nikolaevich Drozhzhinov,

Steklov Mathematical Institute of RAS

Gubkina 8,

119991, Moscow, Russia

E-mail: drozzin@mi.ras.ru

Boris Ivanovich Zavialov,

Steklov Mathematical Institute of RAS

Gubkina 8,

119991, Moscow, Russia

E-mail: bzavial@mi.ras.ru 